\title{
Análise de viabilidade do aproveitamento energético do gás de aterro para a cidade de Cruzeiro [SP]: três diferentes cenários
}

\section{Letícia de Oliveira Silva}

Engenheira Ambiental pela Universidade Federal de Itajubá; Mestranda em Engenharia de Energia pela Universidade Federal de Itajubá. Cruzeiro [SP] Brasil. <letolivsilva@hotmail.com>.

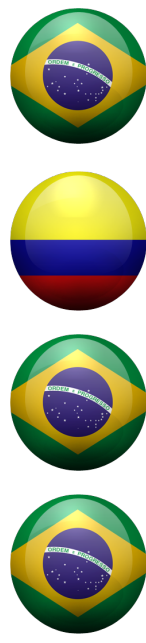

\section{Matheus Martins Lopes}

Engenheiro Ambiental pela Universidade Federal de Alfenas; Especialização em Aplicação das Energias Renováveis pela Universidade Internacional Iberoamericana; Mestrando em Engenharia de Energia pela Universidade Federal de Itajubá. Poços de Caldas [MG] Brasil. <matheusmlopes1@gmail.com>.

\section{Germán Andres Lozano Vela}

Engenheiro Mecânico formado pela Universidad Distrital Francisco Jose de Caldas [Colômbia]; Mestrando em Engenharia de Energia pela Universidade Federal de Itajubá. Bogotá [D.C.] Colômbia.

<gerheavy@hotmail.com>.

\section{Luiz Otávio Campos de Medeiros}

Formado em Ciencias da Computação pela FEPI; Especialização em Eletrônica Industrial pela FUPAI; Mestrando em Engenharia de Energia pela Universidade Federal de Itajubá. Itajubá [MG] Brasil. <lotavio@unifei.edu.br>.

\section{Paulo Antonio de Souza Junior}

Gestor Empresarial pela FATEC; MBA em Administração Financeira pela FACESM; Especialização em Qualidade e Produtividade pela UNIVÁS; Mestrando em Engenharia de Energia pela UNIFEI. Paraisópolis [MG] Brasil. <paulojunior.adm@hotmail.com>.

\section{Resumo}

Os resíduos sólidos estão relacionados a doenças e à poluição ambiental, a necessidade de maior conhecimento da composição desses resíduos e da melhor maneira para a disposição final destes resíduos. A importância do saneamento ambiental pode ser observada na PNRS (Politica Nacional de Resíduos Sólidos), que descreve a necessidade de limpeza urbana e coleta seletiva, a partir da implantação de metas para melhor destinação destes resíduos. Com a finalidade de uma visão geral dos resíduos sólidos produzidos na cidade de Cruzeiro [SP], realizou-se uma projeção e análise da construção de um aterro sanitário para o acondicionamento destes resíduos, iniciando-se pelo ano de 2013, por um período de 20 anos. A falta de lugares para acondicionamento de resíduos resulta em contaminação de lençóis freáticos e solos. Com o auxilio do software "WARM", fez-se a análise de três cenários, com a reciclagem de materiais e utilização para compostagem, sendo os cenários 2 e 3 com o aproveitamento do gás para geração de energia. Após a construção destes cenários foi feita a análise econômica destes cenários. A partir da análise econômica foi possível estabelecer o melhor cenário a ser adotado para a cidade de Cruzeiro [SP].

\section{Palavras-chave}

Projeção populacional, resíduos sólidos, aproveitamento energético.

\section{Power production feasibility analysis from landfill gas in Cruzeiro [SP, Brazil]: three different scenarios}

\begin{abstract}
Solid wastes are related to diseases and environmental pollution, the need for greater knowledge of the waste composition and the best final disposal from this waste. The importance of the environmental sanitation can be observed in PNRS (national solid waste politics), which describes the need for urban cleaning and garbage collection, from the implementation of goals for better allocation of this waste. For the purpose of an overview of the solid waste produced in the city of Cruzeiro [State of Sao Paulo, Brazil], a projection and analysis of the construction of a landfill for the packaging of this waste, beginning by the year 2013, for a period of 20 years. The lack of places for waste packaging results in contamination of groundwater and soils. With the help of the software "WARM", the analysis of three scenarios, with material recycling and composting, and use the 2 and 3 scenarios with the use of gas.
\end{abstract}

\section{Keywords}

Population projection, solid waste, energy recovery. 


\section{Introdução}

A poluição ambiental e o aquecimento global provocam mudanças nas condições do tempo em todas as regiões do mundo e, na maioria dos casos, o fator mais importante é causado pelo aumento da concentração de gases de efeito estufa (Gardiner, 2008). Entre os gases de efeito estufa, o metano é um dos mais importantes (FAO de 2001), devido a ter uma capacidade de cerca de 20 a 30 vezes maior que a do dióxido de carbono na manutenção do calor na atmosfera (Hill \& Kolb, 1999).

Ao se realizar a análise de viabilidade da implantação de uma planta de geração de energia, na qual se captura e incinera o biogás de aterro, deve-se, primeiramente, determinar os aspectos técnicos e econômicos, ou seja, a composição dos resíduos sólidos urbanos, o volume potencial de produção de biogás e o sistema de recuperação e utilização de biogás, o custo da infraestrutura, as oportunidades para a venda de créditos de carbono e da disponibilidade de incentivos e isenções fiscais para este tipo projeto (Álzate \& García, 2009).

Durante muito tempo os resíduos sólidos gerados de vários processos foram classificados apenas como rejeitos e a única preocupação com esses materiais era o acondicionamento distante das cidades; porém, percebeu-se que os destinos para a alocação destes resíduos se tornaram cada vez mais escassos e o acondicionamento sem qualquer preocupação com a geração de subprodutos desses resíduos resultou na contaminação de lençóis freáticos e solos. Após a percepção da relação entre a alocação do lixo e doenças e contaminações, fez-se necessário o maior conhecimento da composição de cada resíduo produzido bem como sua alocação ideal de modo a não prejudicar os recursos naturais.

Segundo Demajorovic (1996), com o interesse nestes resíduos surgiu também o interesse no valor agregado no produto de descartes, bem como o reaproveitamento de alguns materiais. Então se observou a necessidade de uma política de controle de resíduos sólidos, classificando-os e estipulando um destino correto para cada tipo de material.

No Brasil, apesar de mais de $80 \%$ da população estar estabelecida em áreas urbanas (Mansor et al., 2010), a infraestrutura e os serviços básicos não acompanharam o ritmo de crescimento dos centros urbanos; assim, um dos serviços pendentes é o de limpeza urbana e de manejo de resíduos sólidos, e esse déficit impacta na qualidade de vida da população tanto nos centros urbanos quanto nas áreas rurais e periféricas.

Atualmente, com a implantação de metas da Política Nacional de Resíduos Sólidos (PNRS) os resíduos de qualquer natureza são analisados e tratados de maneira diferente do que eram tratados há 50 anos; juntamente com essa política de resíduos sólidos destacou-se também a importância do saneamento ambiental, limpeza urbana e coleta seletiva. Como a política prevê a redução na geração de resíduos, houve a necessidade de se obterem dados sobre geração de lixo em cada cidade, porcentagens de cada tipo de lixo, destino de cada tipo de lixo e a vida útil de cada destinação dos resíduos.

Na PNRS há uma classificação dos resíduos quando à composição, periculosidade, origem e também a relação do destino de cada material; porém, os aterros sanitários são o destino mais comum para o lixo urbano e alguns dos resíduos alocados nesses aterros são perigosos, têm facilidade de combustão ou podem contaminar trabalhadores destes locais; assim, um dos desafios atuais é o manejo adequado dos resíduos, destinando cada componente ao seu local adequado, diminuindo, assim, a quantidade de resíduo descartada incorretamente e desnecessariamente. Aumentando a vida útil de aterros e aperfeiçoando-os, é possível melhorar a qualidade de vida das pessoas que trabalham direta e indiretamente com lixo e evitando a contaminação dos solos e lençóis freáticos.

A norma ABNT NBR 8.419/1992 define aterro sanitário como:

Técnica de disposição de resíduos sólidos urbanos no solo, sem causar danos à saúde pública e à sua segurança, minimizando os impactos ambientais, método este que utiliza princípios de engenharia para confinar os resíduos sólidos à menor área possível e reduzi-los ao menor

(C) Labor \& Engenho, Campinas [SP] Brasil, v.10, n.1, p.07-16, jan./mar. 2016. 
volume permissível, cobrindo-os com uma camada de terra na conclusão de cada jornada de trabalho, ou a intervalos menores, se necessário (ABNT NBR 8.419,1992).

A norma ABNT NBR 8.419/1992 também define o biogás, gás de aterro, ou gás biquímico (GBQ) como sendo uma "mistura de gases produzidos pela ação biológica na matéria orgânica em condições anaeróbias, composta principalmente de dióxido de carbono e metano em composições variáveis".

\section{Material e Métodos}

\subsection{Projeção da população e geração de resíduos}

Para realizar a projeção da população e produção de resíduos sólidos para o município de Cruzeiro [SP], foram coletados dados do IBGE (Instituto Brasileiro de Geografia e Estatística) a respeito da população nos anos de 2000 (Censo Demográfico), 2007 (Contagem Populacional) e 2010 (Censo Demográfico). As populações podem ser observadas na Tabela 1.

Tabela 1. Dados da população de Cruzeiro [SP], e condições a serem atendidas para projeção a partir do modelo do crescimento logístico.

\begin{tabular}{|c|c|c|c|}
\hline \multicolumn{2}{|c|}{ Tempo ( $t_{n}$ anos) } & \multicolumn{2}{|c|}{ População } \\
\hline \multicolumn{2}{|c|}{2000} & \multicolumn{2}{|l|}{73.492} \\
\hline \multicolumn{2}{|c|}{2007} & \multicolumn{2}{|l|}{76.098} \\
\hline \multicolumn{2}{|c|}{2010} & \multicolumn{2}{|l|}{77.039} \\
\hline Condição 1 & $\mathrm{P}_{0}<\mathrm{P}_{1}<\mathrm{P}_{2}$ & $73.492<76.098<77.039$ & Série aceita \\
\hline Condição 2 & $\mathrm{P}_{0 .} \mathrm{P}_{2}<\mathrm{P}_{1}^{2}$ & $5.661 .750 .188<5.790 .905 .604$ & Série aceita \\
\hline
\end{tabular}

Fonte: IBGE, 2015.

Sendo que:

$\boldsymbol{P}_{\boldsymbol{o}}$ - População no ano de 2000; $\mathrm{t}_{0}=2000$.

$\boldsymbol{P}_{1}-$ População no ano de 2007; $\mathrm{t}_{1}=2007$.

$\boldsymbol{P}_{2}$ - População no ano de 2010; $\mathrm{t}_{2}=2010$.

Com estes dados pode-se então proceder ao cálculo da projeção populacional a partir do modelo do crescimento logístico, utilizado neste trabalho (Qasim, 1985 apud Von Sperling, 2005):

$$
P_{1}=\frac{K_{5}}{1+c \cdot e^{a_{l}\left(t-t_{\theta}\right)}}
$$

" $\boldsymbol{P}_{\boldsymbol{t}}$ " refere-se à população estimada do município para os próximos 20 anos, ou seja, " $\boldsymbol{t}$ " irá variar de 2015 a 2035. 0 coeficiente " $K_{s}$ " corresponde à capacidade de saturação da população; " $\boldsymbol{a}_{1}$ " e " $\boldsymbol{c}$ " são os coeficientes que controlam a forma e descrevem a localização da curva, respectivamente; " $\boldsymbol{t}_{\boldsymbol{0}}$ " se refere ao ano 2000; e " $\boldsymbol{t}$ " a cada um dos anos em relação ao período de projeção adotado, no caso de 2015 a 2035.

Os coeficientes " $\boldsymbol{K}_{s}$ ”, “ $\boldsymbol{a}_{\mathbf{1}}$ ” e " $\boldsymbol{c}$ " são encontrados a partir das seguintes equações apresentadas por (Qasim, 1985 apud Von Sperling, 2005):

$$
\begin{gathered}
K_{5}=\frac{2 \cdot P_{0} \cdot P_{1} \cdot P_{2}-P_{1}^{2}\left(P_{0}+P_{2}\right)}{P_{0} \cdot P_{2}-P_{1}^{2}} \\
K_{5}=\frac{2 \cdot 73492 \cdot 76098 \cdot 77039-76098^{2}(73492+77039)}{73492 \cdot 77039-76098^{2}} \Rightarrow K_{5}=77542,9
\end{gathered}
$$




$$
\begin{gathered}
a_{1}=\frac{1}{\left(t_{2}-t_{1}\right)} \cdot \ln \left[\frac{P_{0} \cdot\left(K_{5}-P_{1}\right)}{P_{1} \cdot\left(K_{5}-P_{0}\right)}\right] \\
a_{1}=\frac{1}{(2010-2007)} \cdot \ln \left[\frac{73492 \cdot(77543-76098)}{76098 \cdot(77543-73492)}\right] \Rightarrow a_{1}=-0,355 \\
c=\frac{\left(K_{5}-P_{0}\right)}{P_{0}} \\
c=\frac{(77543-73492)}{73492} \Rightarrow c=0,0055
\end{gathered}
$$

\subsection{Geração de metano e gases de efeito estufa}

Para projeção da geração de metano, utilizou-se do programa LandGEM da USEPA, que utiliza da seguinte equação de primeira ordem para estimar emissões anuais por um período de tempo determinado (USEPA, 2005):

$$
Q_{C H_{d}}=\sum_{i=1}^{n} \sum_{j=0, I}^{I} k \cdot L_{0} \cdot\left(\frac{M_{i}}{10}\right) \cdot e^{-k \cdot t_{i j}}
$$

Onde:

$\boldsymbol{C H}_{4}=$ geração anual de metano no ano do cálculo ( $\left.\mathrm{m}^{3} / \mathrm{ano}\right)$;

$\boldsymbol{i}=$ incremento de tempo de um ano;

$\boldsymbol{j}$ = (ano do cálculo) - (ano inicial de recebimento de resíduos);

$\boldsymbol{n}=$ incremento de tempo de 0,1 ano;

$\boldsymbol{k}=$ taxa de geração de metano (ano-1);

$\boldsymbol{L}_{\boldsymbol{O}}=$ capacidade potencial de geração de metano $\left(\mathrm{m}^{3} / \mathrm{Mg}\right)$;

$\boldsymbol{M}_{\boldsymbol{i}}=$ massa de resíduos aceita no enésimo ano $(\mathrm{Mg})$;

$\boldsymbol{t}_{i j}=$ idade da j-ésima seção de massa de resíduo aceita no enésimo ano (anos em decimais, por exemplo, 3,2 anos).

Os valores dos coeficientes e adotados neste trabalho foram os valores padrão utilizados pelo programa, sendo eles: $170 \mathrm{~m}^{2} / \mathrm{Mg}$; e: $0,05 \mathrm{ano}^{-1}$.

Para a projeção dos gases de efeito estufa, utilizou-se do programa WARM (Waste Reduction Model) da USEPA (United States Environmental Protection Agency). Este programa foi criado para auxiliar organizações e projetos relacionados a resíduos sólidos a reportar voluntariamente reduções de gases de efeito estufa proporcionadas por várias práticas de gestão de resíduos (USEPA, 2015).

O programa calcula as emissões de gases de efeito estufa de cenários base e de práticas alternativas de gestão de resíduos sólidos, como redução a fonte, reciclagem, combustão, compostagem, e uso de aterros. 0 modelo calcula emissões em toneladas métricas de carbono equivalente (MTCE), toneladas métricas de dióxido de carbono equivalente (MTCO2E), e unidades de energia (milhões de BTU) por uma diversa variedade de tipos de materiais normalmente encontrados em resíduos sólidos urbanos (USEPA, 2015).

\section{Resultados e Discussão}

Na Tabela 2 podem ser encontrados os valores da população projetada de 2015 até o ano de 2035, além da geração de resíduos durante o mesmo período. As Figuras 1 e 2 apresentam os gráficos correspondentes. 
Tabela 2. Projeção da população e geração de resíduos para os próximos 20 anos para a cidade de Cruzeiro [SP].

\begin{tabular}{|c|c|c|c|c|c|c|}
\hline Ano & População & $\begin{array}{l}\text { Índice per capta } \\
\text { de resíduos } \\
\text { sólidos, com } \\
\text { acréscimo de } \\
\text { 1\% (Kg/hab.d) }\end{array}$ & $\begin{array}{l}\text { Quantidade } \\
\text { diária coletada de } \\
\text { resíduos Sólidos } \\
\text { urbanos (Kg/d) }\end{array}$ & $\begin{array}{c}\text { Quantidade } \\
\text { anual coletada } \\
\text { de resíduos } \\
\text { Sólidos Urbanos } \\
\text { (Kg/ano) }\end{array}$ & $\begin{array}{c}\text { Quantidade } \\
\text { anual coletada de } \\
\text { resíduos Sólidos } \\
\text { Urbanos (t/ano) }\end{array}$ & $\begin{array}{c}\text { Quantidade } \\
\text { anual acumulada } \\
\text { coletada de } \\
\text { resíduos Sólidos } \\
\text { Urbanos (t/ano) }\end{array}$ \\
\hline 2015 & 77522 & 0,777 & 60245 & 21989728 & 21990 & 21990 \\
\hline 2016 & 77528 & 0,785 & 60853 & 22211401 & 22211 & 44201 \\
\hline 2017 & 77533 & 0,793 & 61465 & 22434771 & 22435 & 66636 \\
\hline 2018 & 77536 & 0,801 & 62082 & 22660009 & 22660 & 89296 \\
\hline 2019 & 77538 & 0,809 & 62704 & 22887239 & 22887 & 112183 \\
\hline 2020 & 77539 & 0,817 & 63333 & 23116558 & 23116 & 135300 \\
\hline 2021 & 77540 & 0,825 & 63967 & 23348039 & 23348 & 158647 \\
\hline 2022 & 77541 & 0,833 & 64608 & 23581743 & 23582 & 182229 \\
\hline 2023 & 77542 & 0,842 & 65254 & 23817719 & 23818 & 206047 \\
\hline 2024 & 77542 & 0,850 & 65907 & 24056008 & 24056 & 230103 \\
\hline 2025 & 77542 & 0,858 & 66566 & 24296647 & 24297 & 254400 \\
\hline 2026 & 77542 & 0,867 & 67232 & 24539670 & 24540 & 278939 \\
\hline 2027 & 77543 & 0,876 & 67904 & 24785107 & 24785 & 303725 \\
\hline 2028 & 77543 & 0,884 & 68584 & 25032986 & 25033 & 328758 \\
\hline 2029 & 77543 & 0,893 & 69269 & 25283336 & 25283 & 354041 \\
\hline 2030 & 77543 & 0,902 & 69962 & 25536184 & 25536 & 379577 \\
\hline 2031 & 77543 & 0,911 & 70662 & 25791555 & 25792 & 405369 \\
\hline 2032 & 77543 & 0,920 & 71368 & 26049478 & 26049 & 431418 \\
\hline 2033 & 77543 & 0,930 & 72082 & 26309978 & 26310 & 457728 \\
\hline 2034 & 77543 & 0,939 & 72803 & 26573081 & 26573 & 484301 \\
\hline 2035 & 77543 & 0,948 & 73531 & 26838814 & 26839 & 511140 \\
\hline
\end{tabular}

\subsection{Projeção do crescimento populacional para cidade de Cruzeiro [SP]}

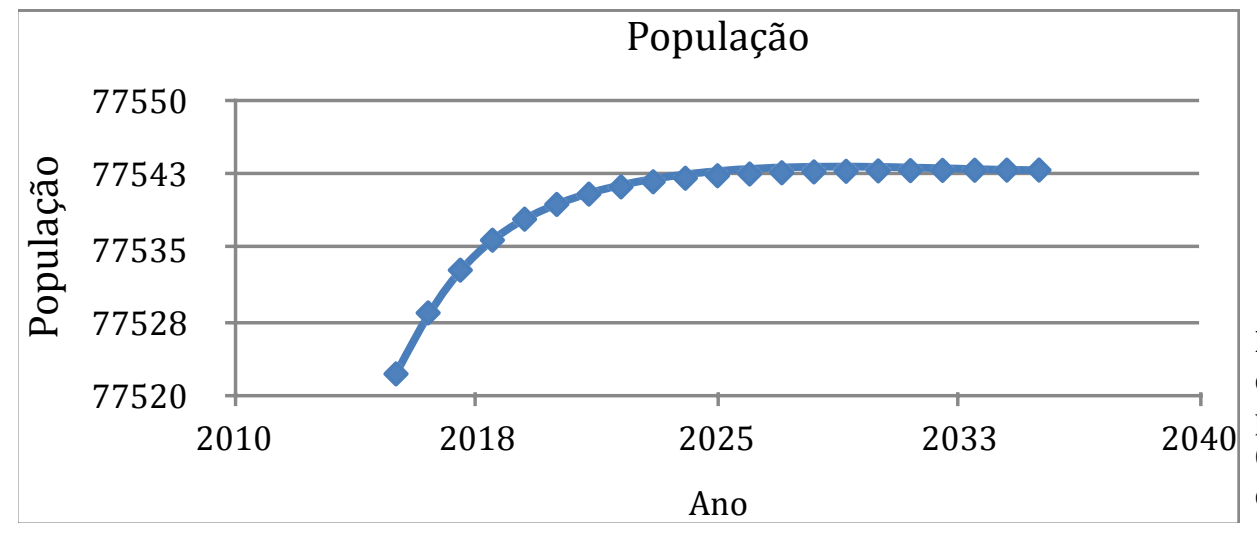

Figura 1. Prognóstico do crescimento populacional para Cruzeiro [SP] até o ano de 2035. 
Nesta projeção, a saturação do crescimento populacional para a cidade seria alcançada no ano de 2027, sendo observado um acréscimo de apenas 21 pessoas em relação ao ano de 2015.

\subsection{Projeção da geração de resíduos sólidos urbanos para a cidade de Cruzeiro [SP]}

Também foi possível obter a partir dos cálculos uma projeção da quantidade anual coletada de resíduos sólidos urbanos no decorrer dos anos (Figura 2).

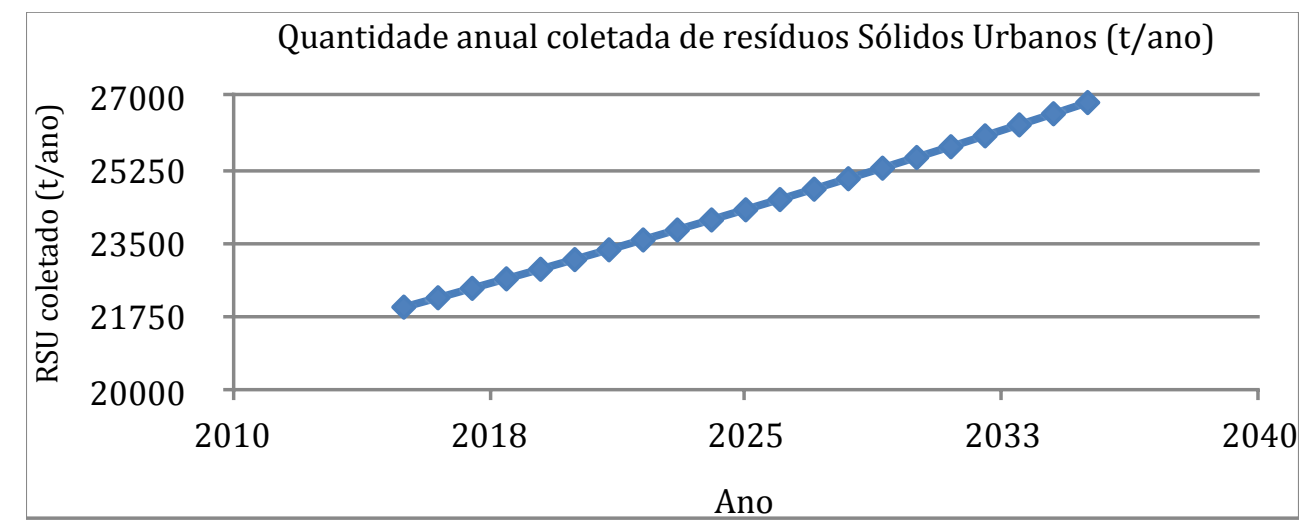

Figura 2. Quantidade anual coletada de resíduos sólidos urbanos, em toneladas, na cidade de Cruzeiro [SP].

Nota-se que a geração de resíduos sólidos aumenta quase que linearmente com o decorrer dos anos, e que, diferentemente da população que para de crescer em 2027, a geração de resíduos sólidos continua aumentando. De acordo com a projeção, o ano de 2035 tem uma quantidade de resíduos coletada aproximadamente $22 \%$ maior que em 2015. Portanto, o aumento da geração de resíduos não acompanha o crescimento populacional da cidade de Cruzeiro [SP], sendo aquele muito maior.

\subsection{Geração de metano}

As Figuras 3 e 4 apresentam a projeção da produção de gases no aterro sanitário para Cruzeiro [SP].

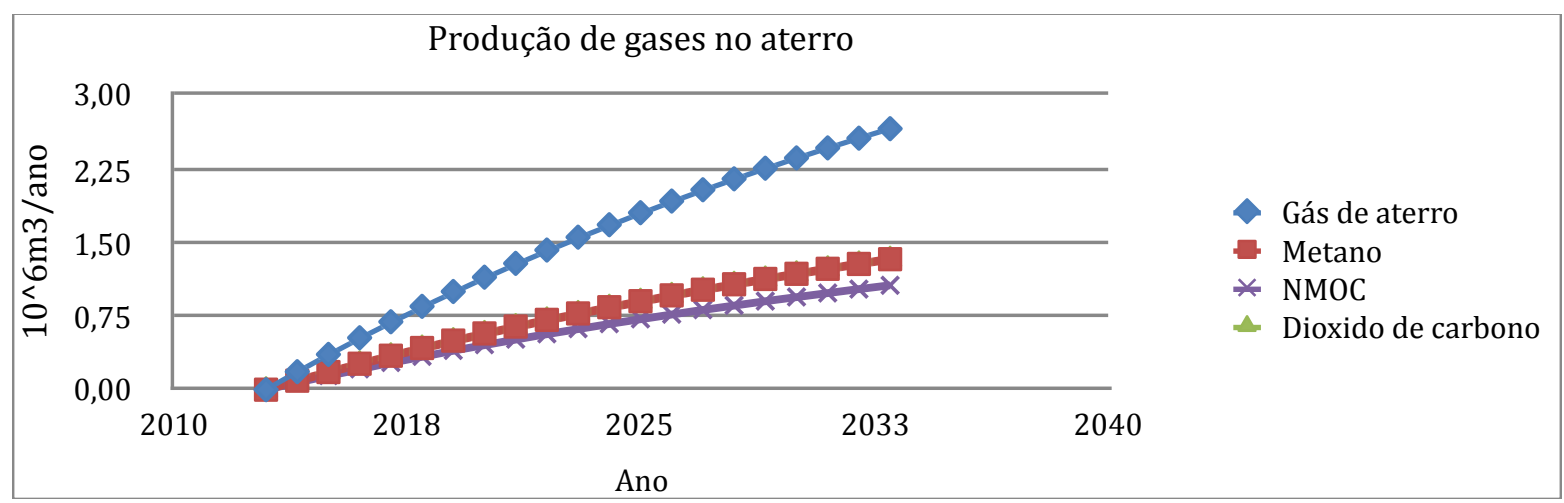

Figura 3. Produção de gases no aterro sanitário de Cruzeiro [SP] até o ano de 2040.

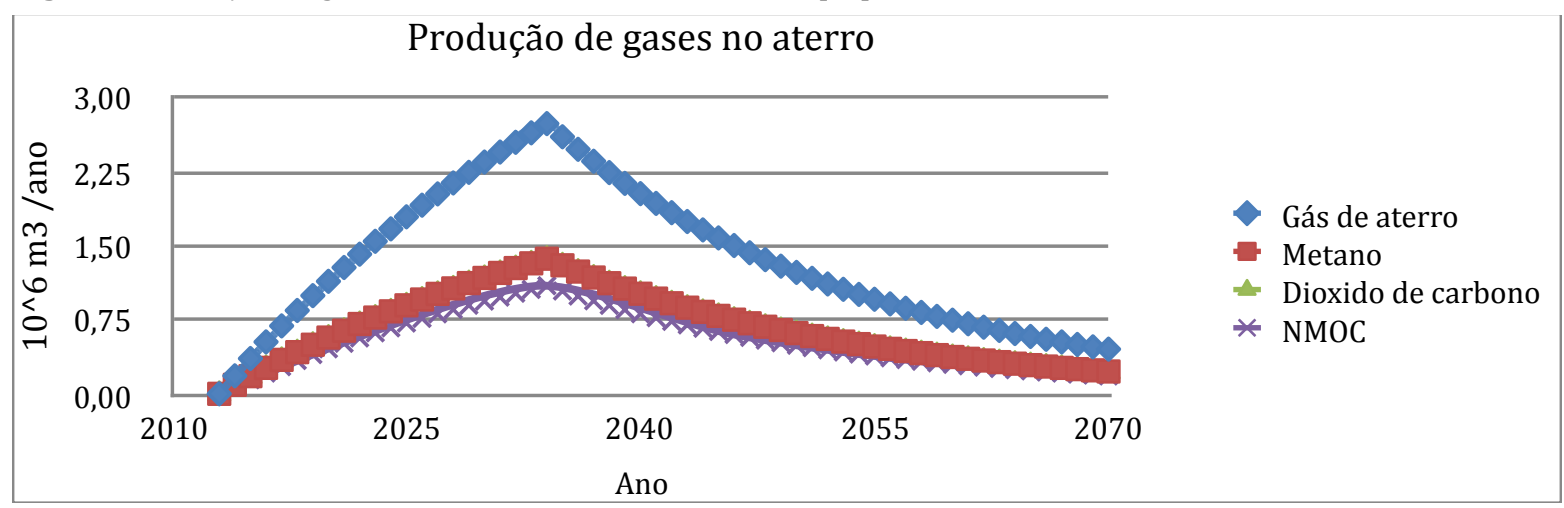

Figura 4. Produção de gases no aterro sanitário de Cruzeiro [SP] até o ano de 2070. 
Percebe-se que o pico de produção de gases é alcançado no ano de 2034, ou seja, um ano antes do encerramento considerado para o aterro, gerando um total de 2,75 x $106 \mathrm{~m}^{3}$ de gás de aterro, dos quais 1,37 x $106 \mathrm{~m}^{3}$ são metano, o gás de maior interesse para conversão energética.

\subsection{Geração de gases de efeito estufa}

Para análise da geração de gases de efeito estufa em relação ao aterro sanitário de Cruzeiro [SP], considerou-se a quantidade de resíduos sólidos gerados no ano de 2015, que totalizou, através dos cálculos realizados anteriormente, 21.990 toneladas. A composição gravimétrica considerada foi a constante no Diagnóstico dos Resíduos Sólidos Urbanos de 2012, do Instituto de Pesquisa Econômica Aplicada (IPEA). A Tabela 3 mostra estes valores e a quantidade de resíduos em relação ao município em estudo.

Tabela 3. Composição gravimétrica e quantidade de resíduos para o município de Cruzeiro [SP].

\begin{tabular}{|ccc|}
\hline Material & Porcentagem & Quantidade (T) \\
\hline Metais & $2,90 \%$ & 637,7 \\
\hline Papel, papelão, tetrapak & $13,10 \%$ & 2880,7 \\
\hline Plástico & $13,50 \%$ & 2968,7 \\
Vidro & $2,40 \%$ & 527,8 \\
\hline Matéria orgânica & $51,40 \%$ & 11302,9 \\
\hline Outros & $16,70 \%$ & 3672,3 \\
\hline Total & $100 \%$ & 21990 \\
\hline
\end{tabular}

Fonte: Adaptado de IPEA, 2012.

Então, três cenários foram analisados utilizando-se do programa WARM (Waste Reduction Model) da USEPA (United States Environmental Protection Agency). No cenário base foi considerada a hipótese de que todos os materiais fossem diretamente apenas para o aterro sanitário, sem reciclagem, redução, compostagem ou incineração. Estes cenários base foram divididos em cenário base A, sem recuperação do gás de aterro, e cenário base $\mathrm{B}$, com recuperação do gás e conversão energética.

O cenário base A foi comparado com um cenário 1, que considerou porcentagens de materiais reciclados baseados em dados do IPEA (2012): para metais em geral, considerou-se uma taxa de reciclagem de $35 \%$; para papel/papelão $45 \%$; para plásticos $20 \%$; e vidros também $20 \%$.

A Tabela 4 apresenta os resultados da simulação do WARM para o cenário base "A" comparado ao cenário 1.

Tabela 4. Resultados da simulação com o WARM para a linha de base "A" e cenário 1.

\begin{tabular}{|lccc|}
\hline & $\begin{array}{c}\text { Emissões totais de GEE } \\
\text { (MTCE) }\end{array}$ & $\begin{array}{c}\text { Emissões totais de GEE } \\
\text { (MTCO2E) }\end{array}$ & $\begin{array}{c}\text { Alteração total no uso energético } \\
\text { (milhões de BTU) }\end{array}$ \\
\hline Cenário base A & 6753 & 24761 & 11598 \\
Cenário 1 & 4613 & 16914 & -54302 \\
\hline
\end{tabular}

Observa-se que, apesar de não haver coleta do gás de aterro neste caso, a reciclagem de parte dos materiais traz benefícios, reduzindo as emissões de gases do efeito estufa e o uso energético.

$\mathrm{O}$ cenário base $\mathrm{B}$ foi comparado com os cenários 2 e 3 . Para o cenário 2 , as porcentagens de reciclados são as mesmas citadas anteriormente, ou seja, são baseados em valores do IPEA (2012).

E um cenário 3 seria o mais otimista, com recuperação do gás de aterro e conversão energética. Além disso, considerou-se redução na fonte de vidro de $20 \%$, com reciclagem passando de $20 \%$ para $40 \%$; 
reciclagem de papéis passando de $45 \%$ para $65 \%$; reciclagem de metais passando de $35 \%$ para 55\%; reciclagem de plásticos passando de $20 \%$ para $40 \%$; e compostagem de $20 \%$ da matéria orgânica.

Na Tabela 5 encontram-se os resultados para o cenário 2 e para o cenário 3, mais otimista, como mencionado anteriormente.

Tabela 5. Resultados da simulação com o WARM para os cenários 2 e 3.

\begin{tabular}{|lccc|}
\hline & $\begin{array}{c}\text { Emissões totais de GEE } \\
\text { (MTCE) }\end{array}$ & $\begin{array}{c}\text { Emissões totais de GEE } \\
\text { (MTCO2E) }\end{array}$ & $\begin{array}{c}\text { Alteração total no uso energético } \\
\text { (milhões de BTU) }\end{array}$ \\
\hline Cenário base B & -496 & -1818 & -2755 \\
Cenário 2 & -1906 & -6990 & -67210 \\
Cenário 3 & -2843 & -10425 & -110935 \\
\hline
\end{tabular}

Observa-se que na Tabela 5, o valor cenário base B é menor que o valor do cenário base A, da Tabela 4. Isso se deve ao fato de que neste primeiro caso considerou-se que não haveria recuperação do gás de aterro, havendo apenas reciclagem de parte dos materiais. Portanto, é visível a importante diferença quando então se passa a também considerar que o gás será recuperado e também utilizado para conversão energética. Também se constata as significativas reduções das emissões do cenário 2 para o cenário 3, o que mostra a importância da redução da produção de resíduos, do aumento da reciclagem de materiais, e também da atividade de compostagem de parte da matéria orgânica.

A Tabela 6 mostra a quantidade de resíduos dispostos em cada técnica de gestão de resíduos sólidos, ou seja, de redução na fonte, materiais reciclados, e disposição em aterro, considerando as porcentagens relacionadas anteriormente.

Tabela 6. Quantidade de resíduos para cada técnica de gestão de resíduos sólidos para os cenários avaliados.

\begin{tabular}{|c|c|ccc|ccc|ccc|}
\hline \multicolumn{7}{|c|}{ Quantidade de resíduos sólidos (t) } \\
\hline \multicolumn{10}{|c|}{ Redução na fonte } & \multicolumn{3}{c|}{ Reciclada } \\
\hline Material & $\begin{array}{c}\text { Cenário } \\
\text { Base (t) }\end{array}$ & Cen. 1 & Cen. 2 & Cen. 3 & Cen. 1 & Cen. 2 & Cen. 3 & Cen. 1 & Cen. 2 & Cen.3 \\
\hline Vidro & 637,7 & 0 & 0 & 105,6 & 105,6 & 105,6 & 211,1 & 422,2 & 422,2 & 211,1 \\
Papéis & 2880,7 & 0 & 0 & 0 & 1296,3 & 1296,3 & 1872,4 & 1584,4 & 1584,4 & 1008,2 \\
Metais & 2968,7 & 0 & 0 & 0 & 223,2 & 223,2 & 350,7 & 414,5 & 414,5 & 287,0 \\
$\begin{array}{c}\text { Plásticos } \\
\text { Matéria } \\
\text { orgânica }\end{array}$ & 527,8 & 0 & 0 & 0 & 593,7 & 593,7 & 1187,5 & 2374,9 & 2374,9 & 1781,2 \\
$\begin{array}{c}\text { Resíduos } \\
\text { sólidos } \\
\text { misturados }\end{array}$ & 11302,9 & 0 & 0 & 0 & - & - & - & 10172,6 & 10172,6 & 8477,1 \\
\hline
\end{tabular}

\subsection{Análise de viabilidade}

Para a análise de viabilidade econômica utilizou-se valores adotados por Leme et al. (2014), descritos na Tabela7.

Para o cálculo considerou-se um prazo de 10 anos e uma TMA de $12 \%$. Nenhum dos três cenários adotados foi economicamente viável devido à baixa potencia obtida e aos altos valores necessários de investimento, mesmo que se considere a redução na quantidade de carbono emitida e os valores obtidos com os créditos de carbono. 
Tabela 7. Valores utilizados para o cálculo da viabilidade econômico da construção do aterro sanitário e aproveitamento energético.

\begin{tabular}{|c|c|c|c|c|}
\hline & Geral & Cenário 1 & Cenário 2 & Cenário 3 \\
\hline \multicolumn{5}{|c|}{ Investimentos } \\
\hline Planta de aproveitamento do gás de aterro & 1.200 .000 US\$/MW & 0 & 225.600 & 225.600 \\
\hline Instalação do aterro & 30.000 US\$/há & 53.499 & 53.499 & 53.499 \\
\hline Serviços (Engenharia, legais, entre outros) & 200.000 US $\$$ & 200.000 & 200.000 & 200.000 \\
\hline Registro do projeto & 100.000 US\$ & 100.000 & 100.000 & 100.000 \\
\hline \multicolumn{5}{|c|}{ Custos e despesas } \\
\hline Manutenção do aterro & $3 \%$ custo do aterro & 2.675 & 1.070 & 1.070 \\
\hline \multirow[t]{2}{*}{ Manutenção do flare } & $2 \%$ custos de instalação & 0 & 4.512 & 4.512 \\
\hline & \multicolumn{4}{|l|}{ Receita } \\
\hline Produção de energia & 17 US\$/MWh & 0 & 27.997 & 27.997 \\
\hline Créditos de carbono & $9 *$ US $\$ /$ tCeq & 62.262 & 38.178 & 76.311 \\
\hline VPL & & -16.824 & -23.6740 & -21.280 \\
\hline
\end{tabular}

*Fonte: Bovespa, 2015.

Dentre os três cenários considerados, aquele que mais se aproxima de ser economicamente viável é o primeiro cenário, sem aproveitamento de energia, com maior valor presente líquido (VPL). Podendo se tornar viável com a valorização dos créditos de carbono, com o preço da tonelada de carbono equivalente acima de 10,4 US\$/t.

\section{Conclusão}

Os cálculos realizados neste trabalho para a cidade de Cruzeiro [SP] possibilitaram avaliar uma série de fatores relacionados aos resíduos sólidos. Inicialmente, a projeção do crescimento populacional para o município em estudo, realizada através do modelo de crescimento logístico, resultou que a saturação deverá ocorrer no ano de 2027, com acréscimo de apenas 21 pessoas em comparação ao ano de 2015, alcançando 77.543 habitantes. Apesar disso, a quantidade de resíduos sólidos urbanos produzidos continuará crescendo a uma taxa maior, passando das 21.990 toneladas em 2015, para 26.839 toneladas em 2035. Isso caracteriza o fenômeno que vem ocorrendo em grandes cidades, onde - mesmo com a estabilização do crescimento populacional - a quantidade de resíduos sólidos gerados continua a crescer, por diversos fatores como, por exemplo, o aumento da renda da população que leva ao aumento da quantidade de resíduos sólidos gerados.

Em relação aos gases produzidos no aterro sanitário da cidade de Cruzeiro [SP], projeta-se que o pico de produção de gases deverá ser alcançado no ano de 2034, um ano antes do considerado para o encerramento do aterro, gerando um total de $2,75 \times 106 \mathrm{~m}^{3}$ de gás de aterro, dos quais 1,37 x $106 \mathrm{~m}^{3}$ são metano, o gás de maior interesse para conversão energética. Contudo, apesar da possibilidade de utilizar este gás para produção de energia, verificou-se por meio das análises econômicas, que a instalação de uma central geradora não seria economicamente viável, mesmo considerando-se a venda de créditos de carbono. A viabilidade talvez pudesse ser alcançada, se houvesse, por exemplo, maiores incentivos governamentais, principalmente para cidades de menor porte; ou ainda, o incentivo a consórcios locais com cidades de uma mesma região. No caso de Cruzeiro [SP], poderia haver um estudo nesse sentido envolvendo as cidades vizinhas de Cachoeira Paulista [SP], Silveiras [SP], e Lavrinhas [SP] entre outras.

A aplicação de uma taxa a ser cobrada dos habitantes locais pela geração de resíduos sólidos poderia estimular a redução da produção desses resíduos, bem como a reciclagem de materiais, o que poderia viabilizar a construção do aterro sanitário, como observa Hortolani (2008), salientando ainda que a PNRS 
prevê que a disposição final ambientalmente adequada é "ordenada de rejeitos em aterros, observando normas operacionais específicas de modo a evitar danos ou riscos à saúde pública e à segurança e a minimizar os impactos ambientais", e dessa forma claramente se mostra a necessidade de criação de aterros sanitários para disposição de resíduos e a necessidade de repasse de incentivos à construção dos mesmos, independentemente da viabilidade econômica.

É possível verificar também, por meio das simulações realizadas com o software WARM, a importância de uma gestão mais efetiva dos resíduos para a diminuição das emissões de gases que geram o efeito estufa. Dentre os principais aspectos que trazem maior benefício para tal fim, pode-se destacar a redução dos resíduos produzidos, a reciclagem de materiais, e a compostagem de matéria orgânica.

\section{Referências}

Álzate, D., \& García, A. Análisis de viabilidad para el aprovechamiento energético de vertederos controlados de residuos sólidos urbanos. (2009). Tese de Doutorado, Universidad Tecnológica de Pereira, Pereira, Colombia.

Bolsa de Valores de São Paulo. (n.d.). <http://www.bmfbovespa.com.br/home.aspx?idioma=pt-br>. Acesso: 9 out. 2015.

FAO (Organização das Nações Unidas para Alimentação e Agricultura, IT). La agricultura, víctima y villano del cambio climático, es la clave del debate sobre cómo entenderlo. (em línea). 2001.

Gardiner, L. (2008). Contaminación atmosférica y cambios climáticos. (em línea). Washington, US, 2008. Disponível em: $<$ http://www.windows.ucar.edu/tour/link=/earth/Atmosphere/pollution_climate_change.sp. html\&edu=high>. Acesso: out. 2015.

Hill, J; \& Kolb, D. (1999). Química para el nuevo milenio (8a ed., 702 p.). México: Prentice Hall.

Hortolani, R. P. S. Análise de viabilidade econômica de um aterro sanitário para cidade de pequeno porte. (2008). Dissertação de Mestrado, Universidade Estadual Paulista "Julio de Mesquita Filho" (Unesp), Ilha Solteira, SP, Brasil.

Instituto Brasileiro de Geografia e Estatística. (n.d.). Infográficos: evolução populacional e pirâmide etária. Disponível em: <http://cidades.ibge.gov.br/painel/populacao.php?lang=\&codmun=351340\&search=sao-paulo/cruzeiro| infogr\%E1ficos:-evolu\%E7\%E3o-populacional-e-pir\%E2mide-et\%E1ria>. Acesso em: out. 2015.

Instituto de Pesquisa Econômica Aplicada. (2012). Diagnóstico dos Resíduos Sólidos Urbanos (relatório de pesquisa). Brasília: Ipea.

Leme, M. M. V., Rocha, M. H., Lora, E. E. S., Venturini, O. J., Lopes, B. M., \& Ferreira, C. H. (2014). Techno-economic analysis and environmental impact assessment of energy recovery from Municipal SolidWaste (MSW) in Brasil. Resources, Conservation and Recycling, 87, 8-20.

Mansor, M. T. C., Camarão, T. C. R. C., Kovacs, M. C., Filet, A. M., Santos, G. A., \& Silva, A. B. (2010). Resíduos sólidos (Série: Cadernos de Educação Ambiental, 6). São Paulo: Governo do Estado de São Paulo, Secretaria do Meio Ambiente. Disponível em: <http://www.ambiente.sp.gov.br/wp-content/uploads/publicacoes/sma/6-ResiduosSolidos.pdfs. Acesso: out. 2015.

NBR 8419: Apresentação de projetos de aterros sanitários de resíduos sólidos urbanos. (1992). Rio de Janeiro: Associação Brasileira de Normas Técnicas.

Sistema Nacional de Informações sobre Saneamento. (2009). Diagnóstico do manejo de resíduos sólidos. Diagnóstico Região Sudeste. Disponível em: < http://www.snis.gov.br/diagnostico-residuos-solidos/diagnosticors-2009>. Acesso em: out. 2015.

United States Environmental Protection Agency. (2015). Waste Reduction Model (WARM). Disponível em: <http://www3.epa.gov/warm/>. Acesso em: nov. 2015.

United States Environmental Protection Agency. (2005). Landfill Gas Emission Model Version 3.02. User'sGuide. Disponível em: <http://www3.epa.gov/ttncatc1/dir1/landgem-v302-guide.pdf>. Acesso em: out. 2015.

Von Sperling, M. (2005). Introdução à qualidade das águas e ao tratamento de esgotos (2a ed. rev., vol. 1, p.60). Belo Horizonte: Universidade Federal de Minas Gerais, Departamento de Engenharia Sanitária e Ambiental. 